\title{
La familia en los tiempos de la diversidad
}

\author{
Family on diversity times \\ Federico Prieto Courries \\ Facultad de Psicología, Universidad Nacional de La Plata, Argentina \\ federicoprietocourries@gmail.com
}

\begin{abstract}
ReSUMEN:
El presente artículo tiene como objetivo indagar, mediante un análisis bibliográfico, la perspectiva epistemológica que nos permita pensar la diversidad y, a partir de ello, construir interrogantes en donde abordar la diversidad en relación con la categoría de familia. En relación a esto, podemos señalar que la distancia con la norma social requiere que los sujetos se vinculen de maneras múltiples, diversas, que incluso no siempre coinciden con las nominaciones que las formas institucionalizadas ofrecen para aportar inteligibilidad a los modos de relacionalidad que habitan por fuera del reconocimiento social. Siendo los modos de relacionalidad sentidos que el sujeto adjudica a los vínculos con otros, a quienes considera significativos y con quienes comparte habitualmente tiempo y actividades. Consideramos indispensable indagar en los sentidos adjudicados a las modalidades de relacionalidad y al modo en que construyen vínculos significativos aquellos sujetos que pertenecen a colectivos socio sexuales disidentes y que por tanto no son regulados por la lógica heteronormativa. En este sentido surgen algunos interrogantes, epodemos hablar de familias diversas? O bien, ¿la perspectiva de la diversidad propone un desmantelamiento de la familia y es necesario pensar en otras configuraciones?
\end{abstract}

Palabras Clave: Familia, Diferencia, Diversidad.

\section{ABstract:}

This article aims to investigate, through a bibliographic analysis, the epistemological perspective that allows us to reflect on diversity and, from that, to construct questions in which to address diversity in relation to the family category. In this case, we can say that the distance with the social norm requires that the subjects are linked to multiple, diverse ways that do not always coincide with the nominations that the institutionalized forms offer to provide intelligibility to the modes of relationality that inhabit the outside of social recognition. Being the modes of relationality, the senses, the subject, the links, the others, those that are considered significant, and those that participate in the time and activities. We consider indispensable in terms of the relations of the relationship and the way to build the bonds of the principles of the collectives. In this sense some questions arise, can we talk about diverse families? Or, does the perspective of diversity propose a dismantling of the family and is it necessary to think of other configurations?

KEYwORDs: Family, Difference, Diversity.

\section{INTRODUCCIÓN}

El presente artículo se propone, mediante un análisis bibliográfico, indagar en la categoría de familia teniendo en cuenta las transformaciones que se han efectuado a lo largo del tiempo e intentando dilucidar cuáles fueron las lógicas que las han sustentado. Luego, se abordará la categoría de diversidad a partir de la construcción de un recorrido epistemológico. Como conclusión, se buscará dar cuenta de algunos interrogantes que pongan en tensión dichas categorías.

El campo desde donde uno se posiciona, es siempre, inevitablemente, un recorte, y ese recorte es consecuencia de decisiones guiadas por el interés y el desinterés, es decir, por aquello que nos interesa porque subsidia el camino hacia las metas de nuestro proyecto histórico como sujetos sociales, y al mismo tiempo, 
sujetos de una actividad disciplinar. Con lo cual toda elección teórica es una elección política (Segato, 2013, p.15).

La ponencia se inscribe en la investigación doctoral denominada "Modos de relacionalidad y construcción de vínculos significativos en sujetos que integran colectivos socio sexuales disidentes”, dirigida por la Psicóloga Edith Pérez y co-dirigida por el Dr. Ariel Martínez.

\section{Aproximaciones a la CATEgoría de FAMilia}

Al considerarse la familia como una institución social, donde confluyen atravesamientos históricos, sociales, culturales y políticos, es posible afirmar que la forma de organización familiar fue cambiando a lo largo de la historia. Es así como pasamos, en el mundo occidental, de la familia ampliada medieval al modelo de familia nuclear. Este tipo de configuración familiar está conformada por dos adultos (varón y mujer) cónyuges y los hijos biológicos, en un número reducido en comparación con la configuración familiar anterior.

Para Lévi-Strauss (1979 [1945]), Héritier (1996) y la antropología estructural en general, la familia es el núcleo básico de alianza y filiación. Y si bien plantean que las formas de organización familiar no siempre fueron las mismas, afirman que siempre hubo una condición ineludible: la estructura familiar debía constituirse en base a la unión hombre-mujer y a los hijos biológicos surgidos de ese encuentro.

Roudinesco (2013), agrega a la posición de Lévi-Strauss de que la universalidad de la familia se basa en la concepción naturalista de la diferencia de los sexos, que es necesaria otra condición para la constitución de la familia: "la existencia previa de otras dos familias, una dispuesta a proporcionar un hombre, y otra, una mujer, que gracias a su matrimonio darán origen a una tercera, y así definitivamente” (2013, p. 14).

Esto nos permite pensar dos enfoques del fenómeno familiar: por un lado, uno sociológico, histórico o psicoanalítico, que hace hincapié en el estudio de las filiaciones y de las generaciones partiendo de las continuidades o discontinuidades entre los padres y los hijos. Y, por otra parte, un enfoque antropológico, que apunta a la descripción horizontal, estructural o comparativa de las alianzas, y que nos dice que cada familia viene de la unión de otras dos familias. En el primer enfoque usaremos la palabra "familia" y, en el segundo caso, hablaremos de "parentesco".

Las relaciones de parentesco, entendidas como la organización social que cada uno de los grupos humanos hace de la reproducción (tanto biológica como social) y por extensión, del control de la sexualidad, no solo se han interpretado desde la antropología como la base de la organización de otros culturales, sino también desde la propia organización de las sociedades occidentales (Pichardo Galan, 2009, p. 28).

Tomando el parentesco como aquella serie de prácticas que establecen relaciones instituyentes de diferentes tipos a partir de las cuales se negocian la reproducción de la vida y las demandas de la muerte, entonces las relaciones de parentesco aparecen para atender a las formas fundamentales de la dependencia humana (Butler, 2006 [2004], p. 150). En esta línea, Butler (2006 [2004]) analiza el debate francés en donde pone en tensión la dimensión del parentesco y del matrimonio intentando vislumbrar cómo el argumento en favor de la alianza legal puede funcionar conjuntamente como una normalización estatal de las relaciones de parentesco reconocibles, una condición que se extiende a los derechos de contrato mientras no perjudique de ninguna forma a las presunciones patrilineales del parentesco (2006 [2004]), p. 152).

Siguiendo a Tajer (2012), es posible afirmar que la configuración familiar -de la familia nuclear-produjo cambios a nivel de lo que hemos denominado organización de la familia pero, a su vez, también lo hizo en torno a los modos en que se relacionan patriarcado y vida familiar. De este modo, los varones pasan a ser pequeños patriarcas de su familia nuclear. Esto ha fortalecido la heterosexualidad en la que se basa la familia nuclear y significó la dominación de varones públicos y mujeres reducidas al mundo de lo privado y a la dimensión sentimental.

Por su parte, Roudinesco (2013) sostiene que la llamada familia conyugal "nuclear" o "restringida", tal como la conocemos hoy en Occidente, es producto de un largo proceso que se extiende del siglo XVI al siglo 
XVIII y que en dicho proceso aquel núcleo al que hace referencia Lévi-Strauss, padre-madre-hijo(s), se separó de lo que conformaba las familias, a saber: un conjunto, una casa, un grupo, que incluía además a los parientes, los allegados, los amigos, los domésticos. Según la autora, esta estructura básica se extendió en Europa desde la Edad Media, mucho antes de constituirse como modelo predominante en la época moderna.

Es así que, encontramos tres grandes momentos en la transformación de la institución familia a lo largo de la historia de occidente. En un primer momento, la llamada familia "tradicional" que apunta fundamentalmente a asegurar la transmisión de un patrimonio. En un segundo momento, la llamada familia "moderna" que se extiende desde fines del siglo XVIII hasta mediados del siglo XX y que se inscribe como un espacio que permite alojar una lógica afectiva que tiene como fundamento el amor romántico, expresado a través del matrimonio en la reciprocidad de sentimientos y deseos carnales. Tomando a Bestard (1998) decimos que la familia moderna se caracterizará por el pasaje a la emoción, lo privado y el individualismo. Y, por último, en la década del sesenta, se presenta la llamada familia "contemporánea" o "posmoderna" que une por un periodo de extensión relativa a dos individuos en busca de relaciones íntimas o expansión sexual (Roudinesco, 2013 [2002], p. 19).

Según Jelin (1998), la familia como orden simbólico y en tanto institución social se encuentra en constante transformación. Los cambios socio-culturales, políticos y la creciente movilización feminista y de la diversidad sexual han cuestionado la familia nuclear como modelo de la familia occidental basado en el vínculo biológico. Este modelo unifica la sexualidad, la procreación y la convivencia a partir del matrimonio heterosexual y monogámico. En cambio, "en términos de organización interna, una familia involucra, según la autora, un conjunto de relaciones: relaciones de producción, de reproducción y de distribución. Dicha organización implica, asimismo, una estructura de poder, y comportamientos afectivos e ideológicos que van reproduciendo tal unidad familiar" (Jelin 2004 en Libson, 2009, p.16).

Este modelo de familia es el que se ha convertido en los últimos tiempos en "La Familia", invisibilizándose la construcción socio-histórica a la que ha respondido dicha configuración. Es decir que, se ha conformado un modelo ideal de familia que funciona como cadena de equivalencia de la "buena familia".

En términos de Habermas (1984), la esfera de lo familiar no está desligada de la esfera económica, por ello es en la esfera de la familia donde históricamente se origina la "privacidad", entendida como interioridad libre y satisfecha (Figari, 2012, p. 24).

Bajo esta concepción de familia, comprobamos que la búsqueda de la universalidad de un tipo de organización familiar responde a la forma en que se organizaron todas las relaciones de parentesco y familia en la cultura occidental. Por ende, la construcción de este modelo de familia como el único válido, es histórica y contextual.

De este modo, y a pesar de las transformaciones socio-históricas que modificaron las relaciones familiares y los vínculos afectivos -llevando a la configuración de distintos tipos de familia (mixtas, adoptivas, de acogida, homoparentales)- podemos decir que el contexto en el que tienen lugar estas formas familiares continúa siendo heteronormativo.

\section{HETERO-NORMA}

La heteronormatividad implica un prejuicio contra la homosexualidad, en el sentido de que incluye al conjunto de normas sociales que ejercen una presión y construyen una sexualidad idealizada, que abarca cuestiones de raza, clase, género y prácticas sexuales.

La heteronormatividad da cuenta de la heterosexualidad como norma obligatoria, universal y natural para la producción e institución de los vínculos amorosos, filiales, familiares. Dicha normativa establece la inteligibilidad cultural de ciertas prácticas y relaciones heterosexuales, excluyendo las no heterosexuales como inimaginables o invisibles. 
Abordar la heterosexualidad significa definir la propia sexualidad en cuanto ésta implica un conjunto de relaciones sociales que son específicamente históricas y culturales y que tienen como matriz la dupla heterosexual-homosexual y hombre-mujer. La sexualidad no es anterior a sus condiciones de producción históricas en el mundo occidental. Cuando Foucault utiliza el término "dispositivo" para referirse a ella, alude a la crítica genealógica, en donde intenta desmontar el efecto ideológico que naturalizó la sexualidad en la modernidad desde un punto de vista etnocéntrico. Desde esta perspectiva, la tecnología del poder que crea los saberes sobre el sexo, a través de la circulación de los discursos y su control, produce una multiplicación y no una exclusión de las sexualidades, clasificándolas en un conjunto de sexualidades periféricas. "Al diseminarlas se trata de sembrarlas en lo real y de incorporarlas al individuo" (Foucault, (2002 [1976]), p. 57-58). De tal manera, siguiendo a con la categorización taxonómica de estas sexualidades periféricas se da un nombre a un deseo ocluido. La ley actúa así en su función represiva que efectivamente produce la heterosexualidad, pero por exclusión-expulsión-repulsa crea al homosexual como otro abyecto. La abyección implica una economía significante que, al mismo tiempo, produce el objeto que niega (Figarí, 2008, p. 97).

Así, cuando aparecen otros deseos desviantes, sea de la heterosexualidad normativa, sea de las sexualidades periféricas, podríamos decir que se constituye otra sexualidad periférica más, en el sentido de que la polisemia discursiva en la lógica de la ontogénesis de lo abyecto es precisamente eso: la "otredad", un significante vacío por contraste siempre ficcionalmente representado desde el universal hegemónico que fija el sentido dominante. Contrastes que sin ser otra cosa comprenden todos los posibles sentidos que la sutura -en su relación de alteridad- estableció, dio nombre y constituyó como diferencia e incluso aquellos posibles sentidos y prácticas que pudieran venir a surgir. La hegemonía, para sustentarse, no puede dejar fisuras, o por lo menos debe intentar controlarlas interpretándolas y reinterpretándolas (Figari, 2008, p. 98).

Butler agrega que la heterosexualidad se impone no solo obligatoriamente, sino también como "comedia inevitable" dada la fuerza y violencia con que se imprime sobre los cuerpos en la pretensión de otorgar una ficción reguladora, es decir, una cadena de equivalencias entre las categorías sexo, género y sexualidad y las de macho $=$ hombre $=$ heterosexual $=$ activo - hembra $=$ mujer $=$ heterosexual=pasiva $(2007$ [1990], p. 265-266).

La radicalidad de considerar la heterosexualidad como matriz fundadora de las relaciones sociales dentro de los análisis históricos, permite no solo evidenciar diferencias entre los sujetos que integran colectivos sexuales disidentes -en razón de su elección sexual- sino que también permite rastrear los mecanismos que sostienen al régimen político que privilegia a la heterosexualidad.

El aporte central del pensamiento de Wittig (2006) radica en identificar una matriz heterosexual desde la cual se designa y construye el sexo de los sujetos, el carácter vincular de las relaciones entre estos y su contingencia dentro del orden heteronormativo. En una suerte de disputa por el ordenamiento que produce el lenguaje, el "pensamiento heterosexual", naturaliza y universaliza los sentidos políticos de la sexualidad, frente a lo cual, Wittig insta a desmontar las categorías que resultan opresivas a través de la creación del lenguaje-manifiesto, lenguaje-acción que transforme y haga historia (2006, p. 56).

De esta manera, Abelleira y Delucca (2011) retoman a pensadores del campo histórico-social como Castoriadis (1975) para hacer referencia a que se asiste a un resquebrajamiento de los valores, mitos, representaciones del conjunto y a un estado crítico y de fragmentación de los imaginarios sociales y de las significaciones sociales que construyen los colectivos en la sociedad. Esta mayor diversidad y fragmentación en las prácticas vinculares producen nuevas formas de establecer vínculos significativos, entendiéndolos como construcciones generadas por el intercambio efectivo entre los miembros que los componen.

Consideramos indispensable entonces, indagar en los sentidos adjudicados a las modalidades de relacionalidad y al modo en que construyen vínculos significativos aquellos sujetos que pertenecen a colectivos socio sexuales disidentes y que por tanto no son regulados por la lógica heteronormativa. 


\section{DE LA DIFERENCIA A LA DIVERSIDAD}

En el dispositivo de la sexualidad, es el varón quien detenta el monopolio del flujo seminal y del placer. La sexualidad, como un dominio separado dentro del campo de la naturaleza humana, la demarcación y el aislamiento conceptual de ese dominio de otras áreas de la vida personal y social, y la generación de identidades sexuales se originan en un primer momento a partir de la oposición entre varón y mujer (Figari, 2012, p. 25).

En Argentina como en el resto de América Latina, el dispositivo de la sexualidad era también un pensamiento reflejo, en líneas generales, de las premisas occidentales del contrato sexual y la caracterización de los sujetos basada en los saberes médicos europeos (Figari, 2012, p. 28).

Como señalamos anteriormente, siguiendo a Foucault, encontramos que en las sociedades modernas de occidente se construyó, a partir del siglo XVIII, un dispositivo denominado dispositivo de la sexualidad, lo que no nos permite decir que éste sustituyó al dispositivo de la alianza. Según el autor, el dispositivo de sexualidad no tiene como razón de ser el hecho de reproducir, sino el de proliferar, innovar, anexar, inventar, penetrar los cuerpos de manera más global. Es decir, entonces, que la sexualidad está ligada a dispositivos de poder recientes y ha estado en expansión creciente desde el siglo XVIII (2002 [1976], p. 103), por lo cual resulta necesario pensar el dispositivo de la sexualidad a partir de las técnicas de poder que le son contemporáneas.

En el campo de las Ciencias Sociales, Rubin (1986 [1975]) utiliza la categoría sistema sexo género para explicar cómo las sociedades organizan la economía -en tanto sistema por el cual los elementos del mundo natural son transformados para el consumo humano- en función de un ordenamiento simbólico de la sexualidad. En tanto, cada sociedad tiene un sistema de sexo género, un conjunto de disposiciones por las cuales el material biológico bruto del sexo y de la procreación es modelado por la intervención humana, social, y satisfecho según convenciones, por extravagantes que puedan ser algunas de ellas" (1986 [1975], p. 97).

Si bien la autora utiliza la categoría de género para el análisis social, la principal crítica que se le ha realizado es que equipara la noción de sexo con la de biología y la de género con la de cultura. Fue a través de los aportes de Foucault (2002[1976]) que se desplomó la adscripción de la sexualidad a la naturaleza. Foucault demuestra que el concepto de sexo no está vinculado a la naturaleza, sino que ha evolucionado históricamente desde el siglo XVIII a través de estudios médicos, demográficos y pedagógicos que construyeron un discurso de verdad con el propósito de normar y disciplinar las conductas sexuales, haciendo de la heterosexualidad el modelo hegemónico.

En la modernidad las sociedades occidentales fueron conformando una experiencia por la que los individuos iban reconociéndose sujetos de su "sexualidad". Pensar en clave de experiencias de dimensión sociohistórica implica poner en consideración la correlación dentro de una cultura entre los campos de saber que se inauguran al respecto, los tipos de normatividad que se establecen, las practicas eróticas y amatorias que se visibilizan y las formas de subjetividad que se construyen (Fernández, 2013, p. 18).

Aparecen hoy identidades diversas, disidentes del modelo heterosexual dominante de la sexualidad. La homosexualidad, el lesbianismo y la transexualidad, son orientaciones que exigen, por una parte, en el marco de los derechos sexuales, el reconocimiento como sujetos de protección en relación con los derechos tradicionales: la no discriminación, la no violencia y la libertad de cambiar la identidad sexual sin perder prerrogativas; y por otra, el derecho a la diferencia, principio político de la posmodernidad que considera a cada individuo particular y libre para ser de la forma que desee, en el contexto de una diversidad práctica y concreta (Salinas, 2006, p. 23).

La aparición tanto en la agenda política y cultural de la sociedad de los movimientos de la disidencia sexual es producto de un largo proceso histórico que aparece en los años setenta y que se extiende hasta nuestros días. El término disidencia sexual alude a pensar la diferencia sexual no en términos de identidades naturalizadas sino como una forma de disenso, entendido no simplemente como habla, sino como una constelación de prácticas, expresiones y creencias no conformistas. Al señalar la presencia de disidencia sexual 
se destaca la existencia de una norma de la cual nos desplazamos o nos alejamos. Desde una política liberal, los conceptos puestos en juego en el escenario político como disidencia sexual o minorías sexuales ocultan que las identidades sexuales y de género son los efectos de una norma que establece los modos adecuados y legítimos de vivir los cuerpos, los placeres y afectos (Flores, 2017, p. 16).

A partir de la segunda mitad del siglo XX, en Occidente varios movimientos sociales -feminismos, blackpower, movimientos de homosexuales, indigenistas, etc.- comenzaron a poner en tensión el binarismo jerárquico que había construido a los varones blancos, cristianos, heterosexuales y propietariosconsumidores. Las denuncias de estos movimientos desnaturalizaban significaciones que habían transformado a lo humano en sinónimo de lo masculino eurocéntrico y de clase (Fernández, 2014, p. 178).

Se gestaron luchas "contra la opresión" de las sexualidades hegemónicas en los espacios públicos y privados que, fundamentalmente, bregaban por la igualdad de oportunidades y por la desnaturalización de las hegemonías del género masculino. Esto ocurrió tanto en el mundo académico -de la mano de los Estudios de la Mujer, los Estudios de Género, los estudios sobre la Masculinidad y los Estudios Queer-, como en el mundo político, con las distintas corrientes feministas y movimientos homosexuales.

En este sentido, en el mundo occidental han sido múltiples las transformaciones que se han efectuado en relación a las cuestiones de género y, al mismo tiempo, se han establecido nuevas problemáticas que es preciso que pensemos. Las transformaciones que se nos presentan en la actualidad no refieren solamente al mundo público laboral, político, cultural, sino que comprenden las prácticas sexuales y la dimensión subjetiva que éstas poseen. En la actualidad, los sujetos rechazan el propio acto de establecer nomenclaturas. Sostienen que se encuentran cómodos con su sexualidad; ésta parece no estar atravesada por culpas y desgarros de generaciones anteriores. Según la autora rechazan la idea de construir identidad sexual, rechazan hacer del rasgo totalidad identitaria y suelen ver, en dicha totalización, totalitarismo (Fernández, 2014, p. 180).

Dichos rechazos son posibles porque previamente se gestaron luchas en los distintos ámbitos y se construyeron espacios en donde fue posible enunciar los reclamos y construir subjetividades dispuestas a visibilizar deseos y pertenencias a colectivos disidentes. Esto les dio mayor visibilidad a travestis, transexuales y transgéneros. Estaríamos en el paso de la diferencia a las diversidades sexuales (Fernández, 2014, p. 180). De todos modos, es imprescindible tener en cuenta que no se trata de un cambio de palabras, sino de la necesidad de construir categorías filosóficas y políticas que sean capaces de comprender y contener dichas transformaciones, con la finalidad de alojar las nuevas manifestaciones.

Tomando al sujeto universal, como idéntico a sí mismo, las configuraciones hegemónicas han circunscripto a todo lo que no es "yo" como el otro, otro que siempre remite a la alteridad, a la diferencia. En tanto el hombre se constituyó como sujeto y el mundo como imagen, en esta producción representadora, este varón blanco, europeo, cristiano, heterosexual y propietario o consumidor será quien establezca todas las normas. Con lo que, "el otro" siempre aparecerá como diferencia, extranjería, complemento o suplemento, un lugar dispuesto para ser ocupado por las mujeres, los homosexuales, las religiones nos hegemónicas. Estos otros se configuran siempre, en palabras de Fernández (2007), como amenazantes y son marcados por dispositivos de discriminación, desigualdad, exclusión y exterminio.

Es decir que para desnaturalizar la amalgama moderna es necesario repensar las condiciones de posibilidad que constituyeron en un mismo movimiento el hombre, el sujeto y la representación. Esto implica considerarlo en términos de producción histórica y no esencial, interrogando lo que la modernidad fundó desde el sujeto y la representación. Recuperando a Deleuze, Fernández (2014) plantea que no se trata de la mera diferencia, sino de la diferencia como lo negativo de lo idéntico, en tanto en el mismo movimiento que se distingue la diferencia, se instituye la desigualdad (2014, p. 183). Nuevamente, no nos encontramos con un mero cambio de palabras, sino que se trata de entender las lógicas que lo sustentan, implica pensar en multiplicidades y no en diferencias, así como también es necesario pensar en producciones de subjetividad y no en sujetos. 
Como fue mencionado anteriormente, hoy nos enfrentamos a una transformación de los imaginarios sociales (de las prácticas, relatos, subjetivaciones, composición de las corporalidades) y de los dispositivos biopolíticos, con sus puntos de resistencia y sus líneas de fuga, lo que genera una reconfiguración de las lógicas colectivas modernas en lo que a la sexualidad respecta (Fernández, 2017, p. 138).

Pensar la diversidad sexual supone problematizar un infinito campo de posibilidades en donde la vivencia del ser sexuado tiene lugar, y no sólo refiere a la pluralidad de orientaciones del deseo y las prácticas sexuales, esto es homosexualidades, bisexualidades, heterosexualidades, transexualidades; sino también que implica convocarnos a re-construir las identidades sexuales que plantean las feminidades y las masculinidades. Preguntarnos sobre la diversidad sexual exige entonces poner en tensión los modelos únicos y aparentemente estáticos que ofrece el sistema de género. Esto nos convoca a indagar en lo complejo y rico que ofrece el campo de expresión de la vivencia de la identidad, de los procesos del cuerpo, los deseos y las prácticas, así como las relaciones posibles que se pueden establecer.

\section{REFLEXIONES FINALES}

Nos encontramos en un momento histórico que nos lleva a replantear ciertas categorías que eran consideradas fijas, fundantes de una sociedad que se sostenía en ellas; pero si pensamos a la diversidad sexual como la referencia a la posibilidad que tienen los sujetos de asumir, expresar y vivir su sexualidad, como también de asumir sus expresiones, preferencias u orientaciones e identidades sexuales, podemos reconocer que todos los cuerpos, todas las sensaciones y todos los deseos tienen derecho a existir y a poder manifestarse.

En relación a esto, es posible afirmar que la distancia con la norma social requiere que los sujetos se vinculen de maneras múltiples, diversas, que incluso no siempre coinciden con las nominaciones que las formas institucionalizadas ofrecen para aportar inteligibilidad a los modos de relacionalidad que habitan por fuera del reconocimiento social. Siendo los modos de relacionalidad sentidos que el sujeto adjudica a los vínculos con otros, a quienes considera significativos y con quienes comparte habitualmente tiempo y actividades.

Se trataría de establecer cuáles son las condiciones de conceptualización necesarias para abordar los nuevos dispositivos de saber-poder y de objetivación-subjetivación en relación a las nuevas formas de dominio y a las nuevas formas de resistencia en lo que refiere a las sexualidades (Fernández, 2013, p. 29).

En este sentido, surgen algunos interrogantes, ¿podemos hablar de familias diversas? O bien, ¿la perspectiva de la diversidad propone un desmantelamiento de la familia nuclear y es necesario pensar en otras configuraciones? En este trabajo, como se señaló en los objetivos, no se pretende responder a estos interrogantes, los cuales emergen a partir de poner en tensión distintos argumentos, no obstante consideramos que nos posibilitan continuar la investigación recuperando las voces de los propios sujetos y sus propias representaciones como sujetos implicados, con el fin de vislumbrar las razones políticas, éticas y pragmáticas que se sostienen dentro del amplio espectro de la diversidad sexual con el fin de obtener voz y visibilidad.

\section{REFERENCIAS BIBLIOGRÁFICAS}

Abelleira, H. y Delucca, N. (2011). Acerca de la familia. En Clínica Forense en Familia, historización de una práctica (pp. 45-63). Buenos Aires: Lugar Editorial.

Bestard, J. (1998). Parentesco y modernidad. Barcelona: Paidós.

Butler, J. (2006 [2004]). Cap. 5. ¿El parentesco es siempre heterosexual de antemano? En Deshacer el género. Barcelona: Ediciones Paidós Ibérica, S.A.

Butler, J. (2007 [1990]) El género en disputa. El feminismo y la subversión de la identidad. Madrid: Paidós. 
Castoriadis, C. (1975). La institución imaginaria de la sociedad, Volumen 1 y 2, Buenos Aires: Tuquets.

Fernández, A. (2007). Las lógicas colectivas. Imaginarios, cuerpos y multiplicidades. Buenos Aires: Editorial Biblos.

Fernández, A. (2014). Las lógicas sexuales: amor, politica y violencias. Buenos Aires: Nueva Visión.

Fernández, A. (2017). Las lógicas sexuales actuales y sus com-posiciones identitarias. En Meler, I. (comp.) Psicoanálisis y género. Escritos sobre el amor, el trabajo, la sexualidad y la violencia. Buenos Aires: Paidós.

Fernández, A y Siqueira Peres, W. (2013). La diferencia desquiciadad. Géneros y diversidades sexuales. Buenos Aires: Editorial Biblos.

Figarí, C. (2008). Heterosexualidades masculinas flexibles. En Jones, D., Figarí, C., Pecheny, M. (comp): Todo sexo es politico. Estudios sobre sexualidades en Argentina. Buenos Aires: Libros El Zorzal.

Figarí, C. (2012). La invención de la sexualidad: el homosexual en la medicina argentina (1880-1930). En Jones, D., Figari, C., Barrón López, S., (comp): La produccion de la sexualidad. Politicas y regulaciones sexuales en Argentina (pp.19-44). Buenos Aires: Editorial Biblos.

Flores, V. (2017). Tropismos de la disidencia. Santiago de Chile: Palinodia.

Foucault, M. (2002[1976]) Historia de la sexualidad 1: la voluntad del saber. Buenos Aires, Siglo Veintiuno Editores.

Héritièr, F. (1996). Masculino/Femenino, El pensamiento de la diferencia. Barcelona: Ariel.

Habermas, J. (1984). Mudança estructural da Esfera Pública: investigações quanto a uma categoría da sociedade burguesa. Rio de Janeiro, Tempo Brasileiro.

Hiller, R. (2012). Regulaciones estatales de la conyugalidad. Aportes sobre el Estado, matrimonio y heteronormatividad. En Jones, D.; Figari, C.; Barrón López, S. (comp). La producción de la sexualidad. Políticas y regulaciones sexuales en Argentina (pp. 89-110). Buenos Aires: Editorial Biblos.

Jelin, E. (1998), Pan y afectos. La transformación de las familias. Buenos Aires: Fondo de Cultura Económica.

Lévi-Strauss, C. (1979 [1945]). Las estructuras elementales del parentesco. Buenos Aires, Paidós.

Libson, M. (2009). La diversidad en las familias: un estudio social sobre parentalidad gay y lesbiana. Tesis de doctorado en Ciencias Sociales, Facultad de Ciencias Sociales, Universidad de Buenos Aires, recuperado de http://inadi.g ob.ar/wp-content/uploads/2010/04/Libson_La-diversidad-en-las-familias.pdf

Meler, I. (2013). Recomenzar: amor y poder después del divorcio. Buenos Aires: Paidos.

Pichardo Galán, J. (2009) Entender la diversidad familiar. Relaciones homosexuales y nuevos modelos de familia. Barcelona: Edicions Bellaterra.

Rich, A. (1996 [1980]). Heterosexualidad obligatoria y existencia lesbiana. Duoda: Revista d'estudis feministes, 10, $15-45$.

Roudinesco, E. (2013). La Familia en desorden. Buenos Aires: Fondo de Cultura Económica.

Rubin, G. (1986 [1975]). El tráfico de mujeres: notas sobre la economía política del sexo. Revista Nueva Antropología, $\operatorname{VIII}(30), 95-145$.

Sánchez Olvera, A. (2009). Cuerpo y sexualidad, un derecho: avatares para su construcción en la diversidad sexual. Sociológica, 24, 69.

Salinas, H. (2006) Políticas públicas de disidencia sexual: apuntes para una agenda. En AA.VV., Disidencia sexual e identidades sexuales y genéricas. México D. F.: Consejo Nacional para Prevenir la Discriminación.

Segato, R. (2013). La crítica a la colonialidad en ocho ensayos, y una antropología por demanda. Buenos Aires: Prometeo Libros.

Tajer, D. (2012) Notas para una práctica psicoanalítica postpatriarcal y posheteronormativa. En Burin M. Meler I, Tajer D. Volnovich J.C. Hazacki Cesar (Comp.) La crisis del patriarcado. Buenos Aires: Topia.

Warner, M. (1991). Fear of a Queer Planet, Social Text, No. 29 (1991), (pp. 3-17). Duke University Press: Stable.

Wittig, M. (2006 [1992]). El pensamiento heterosexual y otros ensayos. Barcelona/Madrid: Egales. 